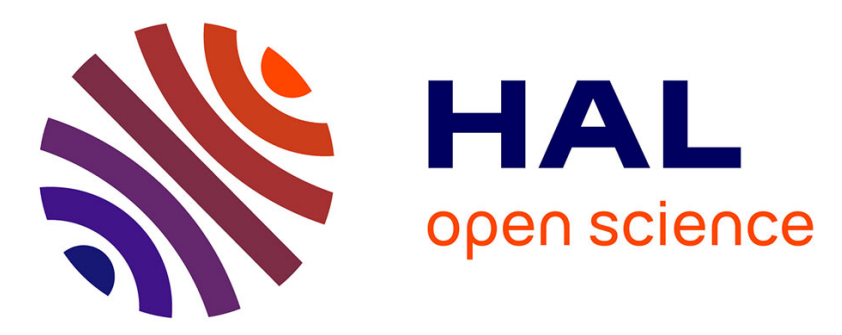

\title{
Salt-induced effective interactions and phase separation of an ultrasoft model of polyelectrolytes
}

Benjamin Rotenberg, Olivier Bernard, J.P. Hansen

\section{To cite this version:}

Benjamin Rotenberg, Olivier Bernard, J.P. Hansen. Salt-induced effective interactions and phase separation of an ultrasoft model of polyelectrolytes. Molecular Physics, 2014, 112, pp.1313. 10.1080/0026897YYxxxxxxxx . hal-01078961

\section{HAL Id: hal-01078961 \\ https: / hal.sorbonne-universite.fr/hal-01078961}

Submitted on 8 Nov 2018

HAL is a multi-disciplinary open access archive for the deposit and dissemination of scientific research documents, whether they are published or not. The documents may come from teaching and research institutions in France or abroad, or from public or private research centers.
L'archive ouverte pluridisciplinaire HAL, est destinée au dépôt et à la diffusion de documents scientifiques de niveau recherche, publiés ou non, émanant des établissements d'enseignement et de recherche français ou étrangers, des laboratoires publics ou privés. 


\title{
Salt-induced effective interactions and phase separation of an ultrasoft model of polyelectrolytes
}

\author{
Benjamin Rotenberg $^{a, b}$, Olivier Bernard ${ }^{a, b}$, Jean-Pierre Hansen $^{a, b, c *}$ \\ ${ }^{a}$ Sorbonne Universités, UPMC Univ Paris 06, UMR 8234 PHENIX, F-75005, Paris, \\ France; \\ ${ }^{b}$ CNRS, UMR 8234 PHENIX, F-75005, Paris, France; \\ ${ }^{c}$ University of Cambridge, Department of Chemistry, Cambridge, UK;
}

(February 12, 2014)

\begin{abstract}
We use a semi-grand canonical version of mean field density functional theory to determine the total effective interaction energy of a solution of penetrable polyions characterized by a gaussian charge distribution, in the presence of added salt. We then apply this effective representation of semi-flexible polyelectrolyte chains to investigate the possibility of a phase separation similar to that predicted earlier for charge-stabilized hard sphere colloids. Apart from the absence of a hard core repulsion, the effective pair potential is similar to the familiar DLVO potential between charged stabilized colloids, i.e. of the screened-Coulomb (Yukawa) form, but the effective valence of the polyions differs significantly from that of the DLVO pair potential, especially at high salt concentration. The existence of a well-defined closed-loop spinodal curve predicted by our mean-field calculation points to a phase separation betweeen solutions with high and low polyion concentrations under reasonable physical conditions. The salt concentration at the upper critical point is typically two orders of magnitude larger than in the case of hard core polyions, indicating that polyion penetrability appears to enhance the tendency towards phase separation.
\end{abstract}

\section{Introduction}

Linear polyelectrolytes are polymer chains carrying anionic or cationic groups which dissociate in water, releasing counterions of opposite charge. A typical example is polystyrene sulfonate which releases $\mathrm{Na}^{+}$or $\mathrm{K}^{+}$cations, leaving a negatively charged polyion. In the limit of low polyion concentrations, the counterions tend to move away from the polyion to maximize their entropy. The highly charged polyion then stretches into an elongated rod-like shape to minimize the electrostatic repulsion between the ionized segments (for reviews, see $[1,2]$ ). At higher concentration, a fraction of the counterions will "condense" onto the charged polyelectrolyte (Manning condensation [3]), thus reducing the electrostatic repulsion between charged segmets, and inducing conformational changes by allowing the polyelectrolyte to fold into a quasi-spherical coil, or a "necklace" of such coils $[1,2]$. Upon adding salt to the polyelectrolyte solution, the electrostatic interactions between segments are increasingly screened, thus enhancing the tendency towards coil-like conformations, as in the case of neutral polymers with short-range interactions between monomers.

Under such conditions it is not unreasonable to adopt a highly coarse-grained description of polyelectrolytes modelled as penetrable spherical objects characterized by a continuous gaussian charge distribution with a width of the order of the

*Corresponding author. Email: jph32@cam.ac.uk 
radius of gyration $R_{g}$ of the coil. Such a representation was recently introduced to investigate solutions of oppositely charged polyelectrolytes, within the so-called "ultrasoft primitive model" (UPM) [4-7]. In the present paper we adapt the model to solutions of equally charged polyelectrolytes in the presence of added salt. We use a classical density functional formulation, similar to that used in Ref. [8], to determine the equilibrium co- and counterion density distributions inside and around the penetrable polyelectrolyte coils, and to calculate the resulting effective pair interactions between "dressed" polyions.

The main objective of these calculations is two-fold. First of all we wish to compare the effective pair potential between penetrable polyions to the well-known DLVO pair potential between charged hard sphere colloids [9]. In the latter case electric double-layers build up near the surface of the charged, inpenetrable colloid, while in the case of penetrable polyions considered here, the co- and counterions spread out over the interior as well as outside the polyelectrolyte coils, so that their local densities are no longer confined outside the polyions, and vary much more smoothly through space. Secondly, we will use the effective interaction between "dressed" polyions to calculate the free energy of the polyion solution, as a function of polyion and salt concentrations, within the random phase approximation (RPA), and explore the possibility of a phase separation between dilute and concentrated polyelectrolyte solutions $[1,2]$. The theory described in the following sections of this paper closely follows a similar calculation for the case of dispersions of charged hard sphere colloids, which indeed predicts a re-entrant phase separation at low salt concentrations [10-12]. The main interest of the present investigation is to analyse how polyion penetrability may affect such a phase separation.

\section{A coarse-grained model of polyelectrolyte coils with added salt}

We consider an aqueous solution of monodisperse polyelectrolyte chains of total charge $Z e$ and monovalent point counterions of charge $-e$ (assuming $Z>0$ without loss of generality), in osmotic equilibrium with a salt reservoir of ideal monovalent co- and counterions. Within the usual "primitive model" of ionic solutions, the polar solvent reduces to a dielectric continuum of permittivity $\epsilon$ (electrostatic units will be used throughout). The polyelectrolyte solution is hence a three-component "mixture" of $n_{1}$ polyions (valence $Z_{1}=Z$ ), $n_{2}$ counterions (valence $Z_{2}=-1$ ) and $n_{3}$ coions (valence $Z_{3}=+1$ ) per unit volume. Overall charge neutrality implies:

$$
\sum_{\alpha=1}^{3} Z_{\alpha} n_{\alpha}=Z n_{1}-n_{2}+n_{3}=0
$$

In the coarse-grained representation $[4,8]$, the polyelectrolyte coils are characterized by a "quenched" gaussian charge distribution of width $\sigma_{1}$ :

$$
\tilde{\rho}_{1}(r)=Z_{1} e \frac{1}{\left(2 \pi \sigma_{1}^{2}\right)^{3 / 2}} e^{-\frac{r^{2}}{2 \sigma_{1}^{2}}}
$$

where $r$ is the distance from the centre of mass position $\mathbf{R}_{i}$ of the $i$ th coil $(1 \leq$ $i \leq N_{1}$ ). A "quenched" (or "frozen") distribution is obviously an approximation since it implies that the internal structure (form factor) of the coil is unaffected by fluctuations of the local charge density of overlapping polyions or microions. The 
latter are assumed to be point ions $\left(\sigma_{2}=\sigma_{3}=0\right)$, such that:

$$
\tilde{\rho}_{\alpha}(r)=Z_{\alpha} e \delta(\mathbf{r}) ; \alpha=2,3 .
$$

The electrostatic potentials generated by these distributions are:

$$
\varphi_{\alpha}(r)=\frac{Z_{\alpha} e}{\epsilon r} \operatorname{erf}\left(\frac{r}{\sqrt{2} \sigma_{\alpha}}\right) ; 1 \leq \alpha \leq 3
$$

and the resulting bare pair potentials between ions are [8]:

$$
v_{\alpha \beta}(r)=\int \varphi_{\alpha}\left(\mathbf{r}^{\prime}\right) \tilde{\rho}_{\beta}\left(\mid \mathbf{r}-\mathbf{r}^{\prime}\right) \mathrm{d} \mathbf{r}^{\prime}=\frac{Z_{\alpha} Z_{\beta} e^{2}}{\epsilon r} \operatorname{erf}\left(\frac{r}{\sqrt{2} \sigma_{\alpha \beta}}\right),
$$

where $\sigma_{\alpha \beta}^{2}=\frac{1}{2}\left(\sigma_{\alpha}^{2}+\sigma_{\beta}^{2}\right)$. Note that the electrosatic repulsion between two polyions and the attraction or repulsion between a polyion and a microion at full overlap $v_{11}(r=0)=u_{11}=Z^{2} e^{2} / \sqrt{\pi} \epsilon \sigma_{11}$ and $v_{12}(r=0)=-v_{13}(r=0)=-Z e^{2} / \sqrt{\pi} \epsilon \sigma_{12}$ are finite, thus preventing the "Coulomb collapse" of polyions and counterions.

The chemical potential $\mu_{2}=\mu_{3}=\mu_{s}$ of the co- and counterions is determined by the salt concentration of the reservoir; if the reservoir ions are assumed to be ideal, their chemical potential $\mu_{s}=k_{B} T \ln \left(n_{s}^{r} \Lambda^{3}\right)$, where $n_{s}^{r}=n_{2}^{r}=n_{3}^{r}$ is the reservoir concentration of anion-cation pairs and $\Lambda$ is the (irrelevant) thermal de Broglie wavelength of the ions.

\section{Density functional theory of the effective interactions between polyions}

The usual strategy for determining the effective interactions between polyions is to trace out the degrees of freedom of the microions for a given configuration $\left\{\mathbf{R}_{i}\right\}$ of the former. Contrary to most previous work based on a canonical description of a closed system of polyions and microions, we adopt a semi-grand canonical point of view which incorporates the equilibrium with the salt reservoir [13, 14]. The independent thermodynamic state variables are hence the total volume of the solution $V$, the temperature $T$, the total number $N_{1}$ of polyions (or equivalently $n_{1}=N_{1} / V$ ) and the salt chemical potential $\mu_{s}$ (or equivalently the reservoir salt concentration $n_{s}^{r}$ ). Accordingly, the total effective interaction between polyions is [15]:

$$
V_{11}^{e f f}\left(\left\{\mathbf{R}_{i}\right\}\right)=V_{11}\left(\left\{\mathbf{R}_{i}\right\}\right)+\Omega\left(\left\{\mathbf{R}_{i}\right\}\right),
$$

where $V_{11}\left(\left\{\mathbf{R}_{i}\right\}\right)=\sum_{i} \sum_{j>i} v_{11}\left(\left|\mathbf{R}_{i}-\mathbf{R}_{j}\right|\right)$ is the bare Coulomb interaction between all polyions (cf. Eq. 5) and $\Omega$ is the equilibrium grand potential of the inhomogeneous fluid of microions in the "external field" due to the polyions located at $\left\{\mathbf{R}_{i}\right\}$; henceforth the dependence of $\Omega$ on $T$ and $V$ will be understood. The grand potential is a functional of the local densities $\rho_{2}(\mathbf{r})$ and $\rho_{3}(\mathbf{r})$ of counterand coions:

$$
\Omega\left[\rho_{2}(\mathbf{r}), \rho_{3}(\mathbf{r}) ; \mu_{s},\left\{\mathbf{R}_{i}\right\}\right]=\mathcal{F}\left[\rho_{2}(\mathbf{r}), \rho_{3}(\mathbf{r}) ;\left\{\mathbf{R}_{i}\right\}\right]-\mu_{s} \sum_{\alpha=2,3} \int \rho_{\alpha}(\mathbf{r}) \mathrm{d} \mathbf{r},
$$

where $\mathcal{F}$ is the free energy functional, which splits into ideal, mean-field and correlation contributions. The latter is neglected within the mean-field framework 
adopted in $[8,10,11]$ as well as here. Moreover the ideal contribution to $\mathcal{F}$ is expanded to second order in the deviations $\Delta \rho_{\alpha}(\mathbf{r})=\rho_{\alpha}(\mathbf{r})-n_{\alpha}$ of the local densities of counterions $(\alpha=2)$ and coions $(\alpha=3)$ from their (yet unknown) mean values $n_{2}$ and $n_{3}$. This approximation is adequate for slowly varying density profiles, a condition that is a priori better satisfied in the present case of penetrable polyions, compared to that of hard core polyions like charge-stabilized colloids examined in [10-12]. The resulting approximate free energy functional reads $[8,10,11]$ :

$$
\begin{aligned}
\mathcal{F}\left[\rho_{2}(\mathbf{r}), \rho_{3}(\mathbf{r}) ;\left\{\mathbf{R}_{i}\right\}\right] & =\sum_{\alpha=2,3}\left\{F_{i d}\left(n_{\alpha}\right)+k_{B} T \ln \left(n_{\alpha} \Lambda^{3}\right) \int \Delta \rho_{\alpha}(\mathbf{r}) \mathrm{d} \mathbf{r}\right. \\
& \left.+\frac{k_{B} T}{2 n_{\alpha}} \int\left[\Delta \rho_{\alpha}(\mathbf{r})\right]^{2} \mathrm{~d} \mathbf{r}\right\}+\frac{e}{2} \int \rho_{c}(\mathbf{r})\left[\psi_{c}(\mathbf{r})+2 \psi_{1}(\mathbf{r})\right] \mathrm{d} \mathbf{r}
\end{aligned}
$$

where $F_{i d}\left(n_{\alpha}\right)=N_{\alpha} k_{B} T\left[\ln \left(n_{\alpha} \Lambda^{3}\right)-1\right], e \rho_{c}(\mathbf{r})=e\left[\rho_{3}(\mathbf{r})-\rho_{2}(\mathbf{r})\right]$ is the local microion charge density, $\psi_{c}(\mathbf{r})$ is the resulting electrostatic potential and $\psi_{1}(\mathbf{r})$ is the electrostatic potential due to the polyions at $\left\{\mathbf{R}_{i}\right\} . \psi_{c}$ satisfies Poisson's equation:

$$
\nabla^{2} \psi_{c}(\mathbf{r})=-\frac{4 \pi e}{\epsilon} \rho_{c}(\mathbf{r})
$$

while $\psi_{1}(\mathbf{r})=\sum_{i=1}^{N_{1}} \varphi_{1}\left(\left|\mathbf{r}-\mathbf{R}_{i}\right|\right)$ with $\varphi_{1}$ defined by Eq. (4). Substituting (8) in Eq. (7), minimization of $\Omega\left[\rho_{2}, \rho_{3}\right]$ with respect to the two density profiles leads to the two coupled Euler-Lagrange equations:

$$
k_{B} T \ln \left(n_{\alpha} \Lambda^{3}\right)+\frac{k_{B} T}{n_{\alpha}} \Delta \rho_{\alpha}(\mathbf{r})+Z e\left[\psi_{c}(\mathbf{r})+\psi_{1}(\mathbf{r})\right]=\mu_{s} ; \alpha=2,3
$$

Two linear combinations of $\rho_{2}(\mathbf{r})$ and $\rho_{3}(\mathbf{r})$, namely $\rho_{c}(\mathbf{r})=\rho_{3}(\mathbf{r})-\rho_{2}(\mathbf{r})$ and $\rho_{X}(\mathbf{r})=x_{2} \rho_{3}(\mathbf{r})+x_{3} \rho_{2}(\mathbf{r})$, with $x_{\alpha}=\frac{n_{\alpha}}{n}\left(n=n_{2}+n_{3}\right)$, decouple the two equations (10) [10, 11], leading to:

$$
\frac{\Delta \rho_{c}(\mathbf{r})}{n}=x_{3} \ln \left(\frac{n_{s}^{r}}{n_{3}}\right)-x_{2} \ln \left(\frac{n_{s}^{r}}{n_{2}}\right)-\beta e\left[\psi_{c}(\mathbf{r})+\psi_{1}(\mathbf{r})\right]
$$

and

$$
\rho_{X}(\mathbf{r})=\frac{n_{2} n_{3}}{n}\left[2+\ln \left(\frac{\left(n_{s}^{r}\right)^{2}}{n_{2} n_{3}}\right)\right]
$$

where $\beta=1 / k_{B} T$ and $n_{s}^{r}$ is the reservoir salt concentration. $\rho_{X}(\mathbf{r})$ is uniform (independent of $\mathbf{r}$ ). The normalization condition:

$$
\frac{1}{V} \int \rho_{X}(\mathbf{r}) \mathrm{d} \mathbf{r}=\frac{1}{V} \int\left[x_{2} \rho_{3}(\mathbf{r})+x_{3} \rho_{2}(\mathbf{r})\right] \mathrm{d} \mathbf{r}=\frac{2 n_{2} n_{3}}{n}
$$

implies, by comparison with Eq. (12), the Donnan equilibrium relation:

$$
n_{2} n_{3}=\left(n_{s}^{r}\right)^{2}=n_{2}^{r} n_{3}^{r}
$$

so that $\rho_{X}(\mathbf{r})$ reduces to $\frac{2 n_{2} n_{3}}{n}$. Combining Eq. (12) with the electroneutrality relation (1) immediately leads to the following result for the counterion and coion 
concentrations in the polyelectrolyte solution:

$$
n_{2}=\frac{1}{2}\left[\sqrt{n_{1}^{2} Z^{2}+4\left(n_{s}^{r}\right)^{2}}+n_{1} Z\right] ; n_{3}=n_{2}-n_{1} Z
$$

Combination of the Fourier transforms of Eqs. (9) and (11) and of $\psi_{1}(\mathbf{r})$ leads immediately to the following solution for the Fourier transform $\hat{\rho}_{c}(\mathbf{k})$ of the microion charge density $\rho_{c}(\mathbf{r})$ :

$$
\hat{\rho}_{c}(\mathbf{k})=\sum_{i=1}^{N_{1}} \hat{\rho}_{c i}(\mathbf{k})=-\sum_{i=1}^{N_{1}} \frac{Z \kappa_{D}^{2}}{k^{2}+\kappa_{D}^{2}} e^{-\frac{k^{2} \sigma_{1}^{2}}{2}} e^{i \mathbf{k} \cdot \mathbf{R}_{i}},
$$

where $\kappa_{D}=\left(\frac{4 \pi n e^{2}}{\epsilon k_{B} T}\right)^{1 / 2}$ is the Debye wavenumber associated with the microions. Inverse Fourier transformation leads to the microion charge density in real space:

$$
\rho_{c}(\mathbf{r})=\sum_{i=1}^{N_{1}} \rho_{c i}(\mathbf{r})=-\sum_{i=1}^{N_{1}} \frac{Z \kappa_{D}^{2}}{4 \pi\left|\mathbf{r}-\mathbf{R}_{i}\right|} f_{2}\left(\left|\mathbf{r}-\mathbf{R}_{i}\right|\right)
$$

where the function $f_{2}(s)$ is defined by:

$$
f_{2}(s)=\frac{1}{2} e^{\kappa_{D}^{2} \sigma_{12}^{2}}\left[e^{-\kappa_{D} s} \operatorname{erfc}\left(\kappa_{D} \sigma_{12}-\frac{s}{2 \sigma_{12}}\right)-e^{\kappa_{D} s} \operatorname{erfc}\left(\kappa_{D} \sigma_{12}+\frac{s}{2 \sigma_{12}}\right)\right] .
$$

$\rho_{c}(\mathbf{r})$ is thus the sum of "orbitals" centered on the $N_{1}$ polyions. The local electrostatic potential within the polyelectrolyte solution $\psi(\mathbf{r})=\psi_{c}(\mathbf{r})+\psi_{1}(\mathbf{r})$ then follows directly via Poisson's equation:

$$
\psi(\mathbf{r})=\sum_{i=1}^{N_{1}} \frac{Z e}{\epsilon\left|\mathbf{r}-\mathbf{R}_{i}\right|} f_{2}\left(\left|\mathbf{r}-\mathbf{R}_{i}\right|\right) .
$$

Comparison of Eqs. (17) and (19) shows that:

$$
\rho_{c}(\mathbf{r})=-\beta \operatorname{en} \psi(\mathbf{r}) .
$$

Note however that Poisson's equation determines the potential $\psi(\mathbf{r})$ only within a constant, say $\psi_{0}$. If $\psi_{0}$ is chosen to be zero in the reservoir, then comparison of Eqs. (20) and (11) shows that they are compatible provided a constant $\psi_{0}$ is added to $\psi(\mathbf{r})$, namely:

$$
\psi_{0}=\frac{k_{B} T}{e}\left[\frac{n_{3}-n_{2}}{n}+\ln \left(\frac{n_{s}^{r}}{n_{3}}\right)\right]
$$

$\psi_{0}>0$ may be identifed with the Donnan potential; as expected $\psi_{0} \rightarrow 0$ as $n_{1} \rightarrow 0$.

The counterion and coion densities can finally be calculated from Eq. (17) and $\rho_{X}=\frac{2 n_{2} n_{3}}{n}$ via the inverse relations $\rho_{2}(\mathbf{r})=\rho_{X}-x_{2} \rho_{c}(\mathbf{r})$ and $\rho_{3}(\mathbf{r})=\rho_{X}+x_{3} \rho_{c}(\mathbf{r})$. Examples of the local densities $\rho_{2 i}(\mathbf{r})$ and $\rho_{3 i}(\mathbf{r})$ around an arbitrary polyion $i$, for two reservoir salt concentrations $n_{s}^{r}$, are shown in Figure 1. Their qualitative behaviour is as expected, with the counterion attracted inside the penetrable polyion, while coions are mostly expelled. At the higher salt concentration the profiles are nearly uniform. 


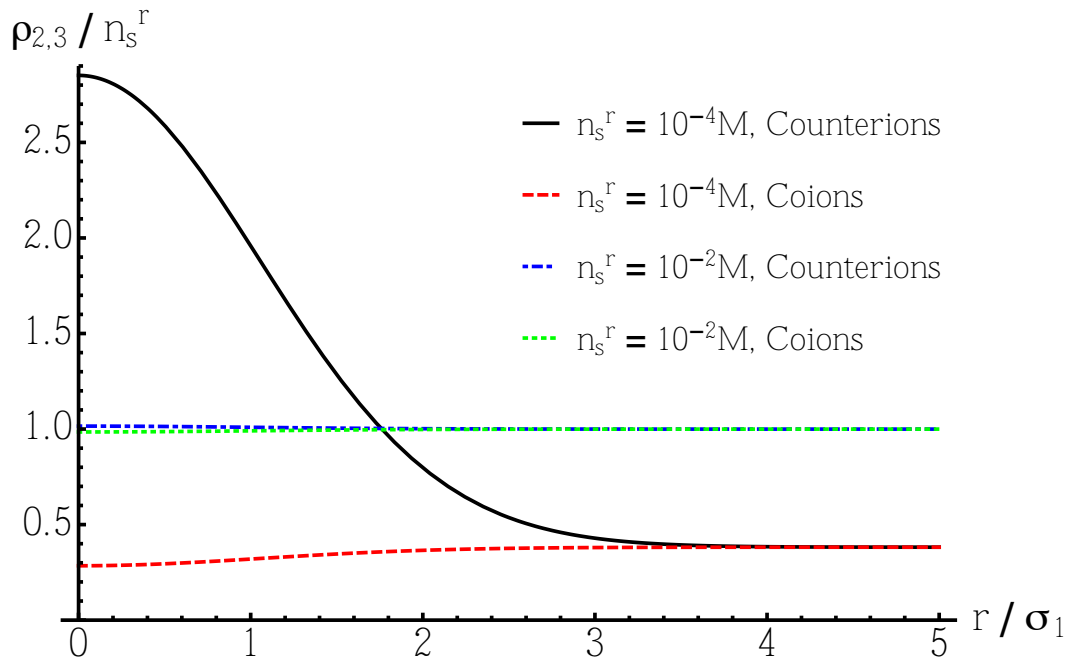

Figure 1. Counterion $\left(\rho_{2}\right)$ and coion $\left(\rho_{3}\right)$ densities around a polyelectrolyte of radius $\sigma_{1}=70 \mathrm{~nm}$ and charge $Z=1000 e$, at a volume fraction $n_{1} \sigma_{1}^{3}=0.1$ in water at room temperature $\left(\beta e^{2} / \epsilon \sigma_{1}=0.01\right)$ and two reservoir salt concentrations $n_{s}^{r}=10^{-4}$ and $10^{-2}$ mol. $\mathrm{L}^{-1}$. In the former case, the deviation from unity of the long-distance limit reflects the Donnan effect.

The equilibrium density profiles and corresponding potentials may now be inserted in the free energy functional (8) to calculate the equilibrium free energy of the microions as a function of polyion coordinates $\left\{\mathbf{R}_{i}\right\}$. The task is greatly simplified by the relation (20) which leads to partial cancellations between the ideal and mean field contributions to the free energy. Gathering results:

$$
\mathcal{F}\left[\rho_{2}, \rho_{3}\right]=F_{i d}\left(n_{2}\right)+F_{i d}\left(n_{3}\right)-\frac{Z N_{1}}{2} k_{B} T \ln \left(\frac{n_{s}^{r}}{n_{3}}\right)+\frac{e}{2} \int \rho_{c}(\mathbf{r}) \psi_{1}(\mathbf{r}) \mathrm{d} \mathbf{r} .
$$

The first three terms are independent of the polyion positions $\left\{\mathbf{R}_{i}\right\}$ and are part of a structure-independent "volume term". The last, Coulomb term may be rewritten as:

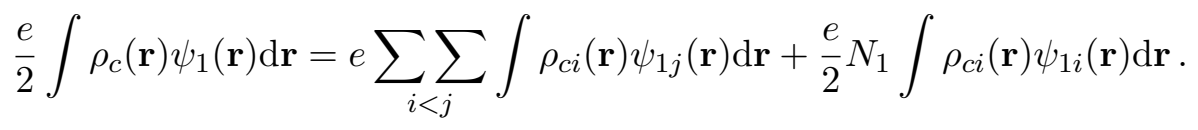

The second term in Eq. (23) is structure-independent and contributes to the volume term. Gathering the results of Eqs. (24), (7), (17), (21), (22) and (23), we arrive at the following expression for the total effective interaction energy between the $N_{1}$ polyions:

$$
V_{11}^{e f f}\left(\left\{\mathbf{R}_{i}\right\}\right)=\sum_{i<j} \sum_{11}^{e f f}\left(\left|\mathbf{R}_{i}-\mathbf{R}_{j}\right|\right)+\mathcal{U}_{0}
$$

The effective pair potential is, setting $r=\left|\mathbf{R}_{i}-\mathbf{R}_{j}\right|$ :

$$
v_{11}^{e f f}(r)=v_{11}(r)+e \int \rho_{c i}\left(\mathbf{r}^{\prime}\right) \psi_{1 j}\left(\left|\mathbf{r}^{\prime}-\mathbf{r}\right|\right) \mathrm{d} \mathbf{r}^{\prime}=\frac{Z^{2} e^{2}}{\epsilon r} f_{2}\left(r ; \sigma_{12} \rightarrow \sigma_{1}\right),
$$

where the function $f_{2}$ is defined in Eq. (18), with $\sigma_{12}$ replaced by $\sigma_{11}=\sigma_{1}$. In the 
limit of point polyions $\sigma_{1} \rightarrow 0, f_{2}\left(r ; \sigma_{12} \rightarrow \sigma_{1}\right)$ simplifies to $e^{-\kappa_{D} r}$, so that $v_{11}^{\text {eff }}(r)$ reduces to the familiar screened Coulomb (or Yukawa) form. For finite polyion size $\sigma_{1}$, the asymptotic behaviour at long distance $r$ is $v_{11}^{e f f}(r) \rightarrow \frac{Z^{2} e^{2}}{\epsilon} e^{\kappa_{D}^{2} \sigma_{1}^{2}} \frac{e^{-\kappa_{D}}}{r}$ [8]. Note that the "apparent valence" of the polyions is $Z e^{\kappa_{D}^{2} \sigma_{1}^{2} / 2}$, which differs from the DLVO apparent valence for hard sphere polyions, namely $Z e^{\kappa_{D} \sigma_{1}} /\left(1+\kappa_{D} \sigma_{1}\right)$, except for $\kappa_{D} \sigma_{1} \ll 1$.

The full volume term $\mathcal{U}_{0}$ in Eq. (24) reads:

$$
\begin{aligned}
\mathcal{U}_{0} & =k_{B} T\left[N_{2} \ln \left(\frac{n_{2}}{n_{s}^{r}}\right)+\left(N_{3}+\frac{Z N_{1}}{2}\right) \ln \left(\frac{n_{3}}{n_{s}^{r}}\right)-\left(N_{2}+N_{3}\right)\right] \\
& -N_{1} \frac{Z^{2} e^{2} \kappa_{D}}{2 \epsilon} \operatorname{erfc}\left(\kappa_{D} \sigma_{1}\right) e^{\kappa_{D}^{2} \sigma_{1}^{2}}
\end{aligned}
$$

Just as its bare counterpart $v_{11}(r)$, the effective pair potential (25) between polyions remains finite as $r \rightarrow 0$ :

$$
v_{11}^{e f f}(r) \stackrel{r \rightarrow 0}{\longrightarrow} \frac{Z^{2} e^{2}}{\sqrt{\pi} \epsilon \sigma_{1}}\left[a\left(\kappa_{D} \sigma_{1}\right)-b\left(\kappa_{D} \sigma_{1}\right)\left(\frac{r}{\sigma_{1}}\right)^{2}+\mathcal{O}\left(r^{4}\right)\right],
$$

where $a(x)=1-\sqrt{\pi} x e^{x^{2}} \operatorname{erfc}(x)$ and $b(x)=\left(1-2 x^{2}\right) / 12+\sqrt{\pi} x^{3} e^{x^{2}} \operatorname{erfc}(x) / 6$. Note that $v_{11}^{e f f}(r=0)$ is strongly reduced in the high salt concentration regime $\kappa_{D} \sigma_{1}>1$, where $a\left(\kappa_{D} \sigma_{1}\right) \ll 1$, while at low salt concentration (weak screening; $\kappa_{D} \sigma_{1} \ll 1$ ) $v_{11}^{e f f}(r)$ goes over to the bare pair potential at small $r\left(a\left(\kappa_{D} \sigma_{1}\right) \rightarrow 1\right)$ [8].

\section{Structure and thermodynamics of the fluid of "dressed polyions"}

The microion degrees of freedom having been traced out, we are now left with a onecomponent system of $N_{1}$ "dressed" polyions whose total interaction energy is given by Eqns. (24)-(26). The pair structure and thermodynamics of this fluid of polyions can now be calculated using the usual tools of liquid state theory [15]. In view of the absence of hard core in the smoothly vayring effective pair potential (25), the simplest approach is based on the random phase approximation (RPA), which has proved successful for systems of penetrable particles, like the gaussian core model [16], provided the reduced amplitude $\beta v(r=0)$ is not too large [7, 17]. In fact, the RPA becomes asymptotically exact in the high density limit of strongly overlapping particles $[18,19]$.

The RPA assumes that the direct correlation function $c(r)$ of the fluid of polyions is given by:

$$
c(r)=-\beta v(r)=-\frac{Z^{2} l_{B}}{r} f_{2}\left(r ; \sigma_{1}\right)
$$

where $l_{B}=\beta e^{2} / \epsilon$ is the familiar Bjerrum length and we have dropped the subscript "11" and the superscript "eff". The corresponding polyion-polyion structure factor follows from the Ornstein-Zernike relation [15]:

$$
S(k)=1+n_{1} \hat{h}(k)=\frac{1}{1-n_{1} \hat{c}(k)}=\frac{k^{2}+\kappa_{D}^{2}}{k^{2}+\kappa_{D}^{2}+\kappa_{D 1}^{2} e^{-k^{2} \sigma_{1}^{2}}},
$$

where $\kappa_{D 1}^{2}=4 \pi Z^{2} l_{B} n_{1}$. The excess free energy of the fluid of polyions due to the 
pair interactions may be calculated via Kirkwood's coupling constant integration method [20] for fixed values of $n_{1}, n_{s}^{r}$ and $T$ :

$$
\begin{aligned}
\frac{\beta F_{1}^{e x}}{N_{1}} & =\frac{n_{1}}{2} \int_{0}^{1} \mathrm{~d} \lambda \int[1+h(r ; \lambda)] \beta v(r) 4 \pi r^{2} \mathrm{~d} r \\
& =\frac{n_{1}}{2} \frac{1}{(2 \pi)^{3}} \int_{0}^{1} \mathrm{~d} \lambda \int_{0}^{\infty} \hat{h}(k ; \lambda) \beta \hat{v}(k) 4 \pi k^{2} \mathrm{~d} k+\frac{n_{1}}{2} \beta \hat{v}(k=0)
\end{aligned}
$$

Using again the RPA ansatz $\hat{c}(k ; \lambda)=-\lambda \beta \hat{c}(k)$ and the Orstein-Zernike relation to determine $\hat{h}(k ; \lambda)$ one arrives, after a trivial integration over the coupling constant $\lambda$, at the following expression:

$$
\begin{aligned}
\frac{\beta F_{1}^{e x}}{N_{1}} & =\frac{\kappa_{D 1}^{2}}{2 \kappa_{D}^{2}}-\frac{\kappa_{D 1}^{2}}{8 \pi^{3 / 2} n_{1} \sigma_{1}}+\frac{\kappa_{D 1}^{2} \kappa_{D}}{8 \pi n_{1}} e^{\kappa_{D}^{2} \sigma_{1}^{2}} \operatorname{erfc}\left(\kappa_{D} \sigma_{1}\right) \\
& -\frac{1}{4 \pi^{2} n_{1}} \int_{0}^{\infty} \ln \left[\frac{k^{2}+\kappa_{D}^{2}}{k^{2}+\kappa_{D}^{2}+\kappa_{D 1}^{2} e^{-k^{2} \sigma_{1}^{2}}}\right] k^{2} \mathrm{~d} k
\end{aligned}
$$

The total free energy $F$ per polyion of the solution follows by adding the ideal term and the volume term $\mathcal{U}_{0}$ defined in Eqn. (26). The last term in the latter exactly cancels the third term in (31). Defining the dimensionless free energy per unit volume $\varphi=\beta F \sigma_{1}^{3} / V$ and introducing dimensionless variables $q=k \sigma_{1} ; q_{D}=\kappa_{D} \sigma_{1}$; $q_{D 1}=\kappa_{D 1} \sigma_{1}$ and $n_{\alpha} \equiv n_{\alpha} \sigma_{1}^{3}$, the complete expression for $\varphi$ reads:

$$
\begin{aligned}
\varphi & =n_{1}\left[\ln n_{1}-1\right]+n_{1} \frac{q_{D 1}^{2}}{2 q_{D}^{2}}-\frac{q_{D 1}^{2}}{8 \pi^{3 / 2}}-\frac{1}{4 \pi^{2}} \int_{0}^{\infty} \ln \left[\frac{q^{2}+q_{D}^{2}}{q^{2}+q_{D}^{2}+q_{D 1}^{2} e^{-q^{2}}}\right] q^{2} \mathrm{~d} q \\
& +\frac{Z n_{1}}{2} \ln \left(\frac{n_{s}^{r}}{n_{3}}\right)-\sqrt{n_{1}^{2} Z^{2}+4\left(n_{s}^{r}\right)^{2}}
\end{aligned}
$$

We recall that $n_{2}$ and $n_{3}$ are determined by $n_{1}$ and $n_{s}^{r}$ via Eqn. (14).

For fixed values of $T$ and $V, \varphi$ is a function of $n_{1}$ and $\mu_{s}$ (or $n_{s}^{r}$ ); it is the thermodynamic potential associated with the semi-grand canonical description of the polyion-salt system adopted from the start. The chemical potential of the polyions follows from the thermodynamic relation:

$$
\beta \mu_{1}=\left(\frac{\partial \varphi\left(n_{1}, \mu_{s}\right)}{\partial n_{1}}\right)_{\mu_{s}} .
$$

The total grand potential per unit volume of the solution, $\omega\left(\mu_{1}, \mu_{s}\right)$, then follows from the Legendre transformation:

$$
\omega\left(\mu_{1}, \mu_{s}\right) \equiv f\left(n_{1}, \mu_{s}\right)-n_{1} \mu_{1}
$$

where $f=F / V=k_{B} T \varphi / \sigma_{1}^{3}$. Remembering that the osmotic pressure $P=-\omega$, we arrive at the following expression for the dimensionless osmotic pressure $p=\beta P \sigma_{1}^{3}$ :

$$
p=n_{1} \beta \mu_{1}-\varphi\left(n_{1}, \mu_{s}\right) .
$$




\section{Polyion phase separation?}

We now address the question of a possible demixing of the polyelectrolyte solution into a dilute ("gas") and a concentrated ("liquid") phase for fixed chemical potential $\mu_{s}$ of the salt. Such a phase separation has been predicted to occur in dispersions of charged hard sphere colloids at very low salt concentrations [10-12] and there is some experimental evidence for such a phase transition [21, 22]. The important question is whether a similar phase separation occurs in the case of penetrable polyions, i.e. in the absence of a hard core repulsion. Phase separation occurs when a plot of the free energy function $\varphi\left(n_{1}, \mu_{s}\right)$ versus polyion density $n_{1}$ at fixed salt chemical potential $\mu_{s}$ exhibits a concave region, corresponding to metastable or unstable thermodynamic states; these are eliminated by the usual Maxwell double-tangent construction which determines the densities $n_{1}^{g}$ and $n_{1}^{l}$ of the coexisting "gas" and "liquid" phases. According to Eqn. (33), the slope of the double-tangent determines the common chemical potential $\mu_{1}$ of the coexisting phases.

In a first instance we have addressed the simpler problem of locating the spinodal line, i.e. the locus of points in the $\left(n_{1}, \mu_{s}\right)$ plane where the curvature:

$$
\xi\left(n_{1}, \mu_{s}\right)=\left(\frac{\partial^{2} \varphi\left(n_{1}, \mu_{s}\right)}{\partial n_{1}^{2}}\right)_{\mu_{s}}
$$

vanishes. The spinodal separates regions of positive and negative curvature of the free energy function, corresponding respectively to the stable or metastable states, and the thermodynamically unstable states. The spinodal and binodal (coexistence) curves meet at the critical point, or at an upper and a lower critical point in the case of closed spinodal curves corresponding to re-entrant phase behaviours (for example see references [10, 12, 23-26]).

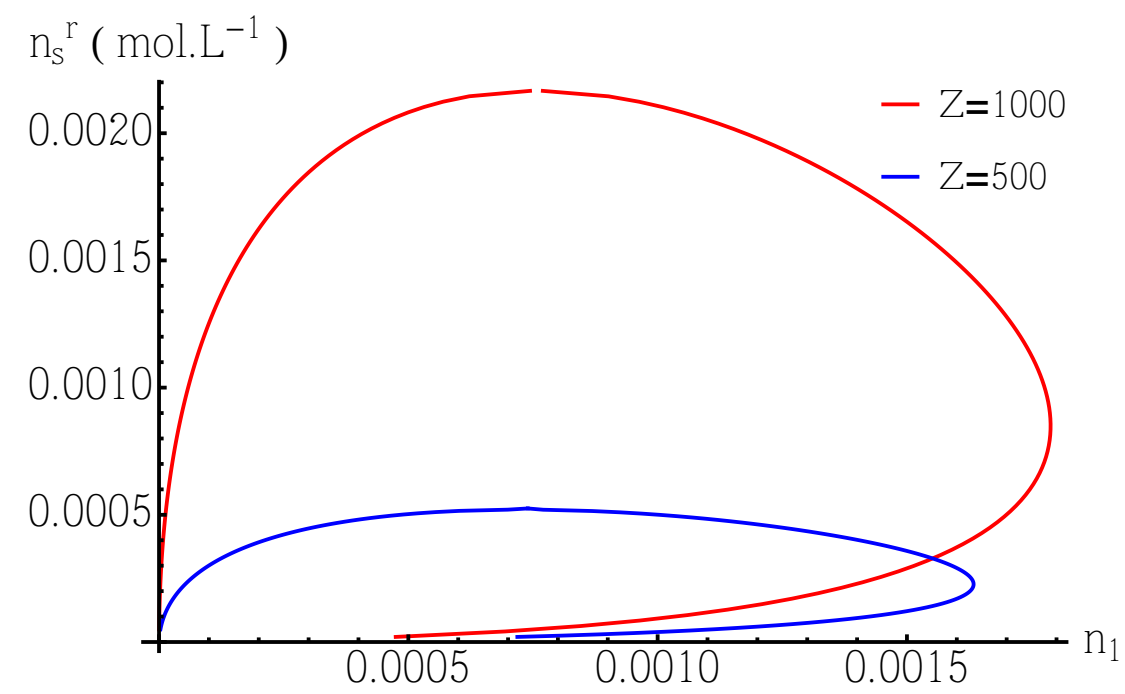

Figure 2. Spinodal curve for a polyelectrolyte of radius $\sigma_{1}=70 \mathrm{~nm}$ with charge $Z=500 e$ (blue) or $1000 e$ (red) in water at room temperature $\left(\beta e^{2} / \epsilon \sigma_{1}=0.01\right)$.

Fig. 2 shows the spinodal in the $\left(n_{1}, n_{s}^{r}\right)$ plane, calculated for $Z=1000$ and $\sigma_{1}=70 \mathrm{~nm}$. It is re-entrant, as in the case of charged hard-sphere colloids [10, 12], i.e. the spinodal forms a closed loop surrounding the region of thermodynamically 
unstable states. The upper critical point occurs at $n_{1} \sigma_{1}^{3} \approx 0.00075$ and a reservoir salt concentration $n_{s}^{r} \approx 2.2 \mathrm{mM}$. The latter value is about two orders of magnitude larger than typical values found in the hard core case $[10,11]$. On the other hand the reduced polyion concentration $n_{1} \sigma_{1}^{3}$ at the critical point is significantly lower than for hard core polyions, presumably as a consequence of polyion penetrability. The lower critical point occurs at extremely low values of $n_{s}^{r}$ and $n_{1}\left(n_{s}^{r} \approx 210^{-11} \mathrm{M}\right.$ and $n_{1} \approx 10^{-11}$ ), which are physically irrelevant. Fig. 2 also shows the spinodal for $\sigma_{1}=70 \mathrm{~nm}$ and the lower valence $Z=500$ (corresponding to a weakly ionized polyelectrolyte). The critical reservoir salt concentration $n_{s}^{r}$ is found to scale roughly like $Z^{2}$, while the critical polyion density is practically unchanged.

\section{Conclusion}

We have used a semi-grand canonical version of mean field density functional theory to determine the total effective interaction energy of a solution of penetrable polyions characterized by a gaussian charge distribution, in the presence of added salt. We then applied this effective representation of semi-flexible polyelectrolyte chains to investigate the possibility of a phase separation similar to that predicted earlier for charge-stabilized hard sphere colloids. It is worth stressing that our ultrasoft polyelectrolyte model involves only purely Coulombic interactions between polyions and microions alike. Our main conclusions can be summarized as follows.

a) Apart from the absence of a hard core repulsion, the effective pair potential is similar to the familiar DLVO potential between charged stabilized colloids, i.e. of the screened-Coulomb (Yukawa) form, but the effective valence of the polyions differs significantly from that of the DLVO pair potential, especially at high salt concentration.

b) The existence of a well-defined closed-loop spinodal curve predicted by our mean-field calculation points to a phase separation betweeen solutions with high and low polyion concentrations under reasonable physical conditions. The salt concentration at the upper critical point is typically two orders of magnitude larger than in the case of hard core polyions, indicating that polyion penetrability appears to enhance the tendency towards phase separation.

A brief critique of the shortcomings of the model used and of the approximations made in the present work is in order. The model assumes a "quenched" charge distribution, which is clearly a gross over-simplification. In real polyelectrolyte solutions the internal structure and charge distribution of the polyions responds to variations of physical conditions, which induce significant changes of the polyelectrolyte conformations. Account must be taken of counterion "condensation" [3] and of the dependence of the electrostatic persistence length on the Debye screening length $[1,2]$. Within the highly coarse-grained description adopted in this paper, at the very least the polyion charge $Z e$ and size $\sigma_{1}$ should be made dependent on salt concentration.

As regards the approximations made in the present work, the RPA used in section 4 leads to the analytic expression (32) for the free energy which requires only a simple quadrature for numerical calculations. At very low salt concentrations the reduced value of the effective pair potential at full overlap $\beta v_{11}(r=0)$ can be larger than $10^{2}$, which is too high to ensure the RPA to be quantitatively reliable. Although our predictions are expected to be at least qualitatively valid, since in the relevant range of low polyion concentrations considered here the probability of two polyions to overlap is very low, systematic corrections to the RPA will be included in future work which will focus on explicit calculations of the phase coexistence 
curve.

\section{References}

[1] J.L. Barrat and J.F. Joanny, Theory of Polyelectrolyte Solutions, in Advances in Chemical Physics, edited by I. Prigogine and Stuart A. Rice (John Wiley \& Sons, Inc., New York, 2007), pp. 1-66.

[2] A.V. Dobrynin and M. Rubinstein, Progress in Polymer Science 30 (11), 1049 (2005).

[3] G.S. Manning, The Journal of Chemical Physics 51 (3), 924 (1969).

[4] D. Coslovich, J.P. Hansen and G. Kahl, The Journal of Chemical Physics 134 (24), 244514 (2011).

[5] D. Coslovich, J.P. Hansen and G. Kahl, Soft Matter 7 (5), 1690 (2011).

[6] A. Nikoubashman, J.P. Hansen and G. Kahl, The Journal of Chemical Physics 137 (9), 094905 (2012).

[7] P.B. Warren, A. Vlasov, L. Anton and A.J. Masters, The Journal of Chemical Physics 138 (20), 204907 (2013).

[8] J.P. Hansen, D. Coslovich and G. Kahl, Molecular Physics 109 (23-24), 2953 (2011).

[9] J.N. Israelachvili, Intermolecular and Surface Forces, 2nd ed. (Academic Press, London, 1992).

[10] R. van Roij, M. Dijkstra and J.P. Hansen, Physical Review E 59 (2), 2010 (1999).

[11] R. van Roij and R. Evans, Journal of Physics: Condensed Matter 11 (50), 10047 (1999).

[12] J.F. Dufrêche, T.O. White and J.P. Hansen, Molecular Physics 101 (11), 1741 (2003).

[13] B. Zoetekouw and R. van Roij, Physical Review E 73 (2), 021403 (2006).

[14] B. Zoetekouw and R. van Roij, Physical Review Letters 97 (25), 258302 (2006).

[15] J.P. Hansen and I.R. McDonald, Theory of Simple Liquids, 4th ed. (Academic Press, London, 2013).

[16] F.H. Stillinger, The Journal of Chemical Physics 65 (10), 3968 (1976).

[17] A.A. Louis, P.G. Bolhuis and J.P. Hansen, Physical Review E 62 (6), 7961 (2000).

[18] A. Lang, C. Likos, M. Watzlawek and H. Löwen, Journal of Physics: Condensed Matter 12 (24), 5087 (2000).

[19] C.N. Likos, Physics Reports 348 (4-5), 267 (2001).

[20] J.G. Kirkwood, The Journal of Chemical Physics 3 (5), 300 (1935).

[21] B.V.R. Tata, M. Rajalakshmi and A.K. Arora, Physical Review Letters 69 (26), 3778 (1992).

[22] K. Ito, H. Yoshida and N. Ise, Science 263 (5143), 66 (1994).

[23] P.B. Warren, The Journal of Chemical Physics 112 (10), 4683 (2000).

[24] A.R. Denton, Physical Review E 73 (4), 041407 (2006).

[25] P.B. Warren, Physical Review E 73 (1), 011411 (2006).

[26] A.R. Denton, Physical Review E 76 (5), 051401 (2007). 